bition: a novel therapeutic strategy for diabetic microangiopathy. Diabetes Vase Dis. 2006:3:172-178.

9. Cavaletti G, Miloso M, Nicolini G, Scuteri A, Tredici G. Emerging role of mitogen-activated protein kinases in peripheral neuropathies. J Periplt Nerv Syst. 2007; 12:175-194.

10. Hall K, Liu KJ, Sima AA, Wiley JW. Impaired inhibitory G-protein function contributes to increased calcium currents in rats with diabetic neuropathy. $J$ Neurophysiol. 2001 ;86:760-770.

11. Varkonyi T. Putz Z, Keresztcs K. Martos T. Lengyel C, Stirban A, Jermendy G, Kempler P. Current options and perspectives in the treatment of diabetic neuropathy. Curr Pltarin Des. 2013;19(27):4981-5007.

12. Boulton AJ, Kempler P, Ametov A, Ziegler D. Whither pathogenetic treatments for diabetic polyneuropathy? Diabetes Metal) Res Rev. 2013;29(5):327-33.

13. Ziegler D. Thioctic acid for patients with symptomatic diabetic polyneuropathy: a critical review. Treat Endocrinol. 2004;3(3): 173-89.

14. Tiaka EK, Papanas N, Manolakis AC, Maltezos E. The role of nerve growth factor in the prophylaxis and treatment of diabetic foot ulcers, hit J Burns Trauma. 2011; 1 (I ):68-76.

15. Bril V, Hirose T, Toniioka S, Buchanan R; Ranirestat Study Group. Ranirestat for the management of diabetic sensorimotor polyneuropathy. Diabetes Care. 2009:32(7): 1256-60.

16. Schcmtnel KE, Padiyara RS, D‘SouzaJJ. Aldose reductase inhibitors in the treatment of diabetic peripheral neuropathy: a review ../Diabetes Complications. 2010;24(5):354-60.

17. Bansal D, Badhan Y, Gudala K, Schilano F. Ruboxistaurin for the Treatment of Diabetic Peripheral Neuropathy: A Systematic Review of Randomized Clinical Trials. Diabetes Metab J. 2013;37(5):375-384.

18. Rajamani K, Colman PG, Li LP, Best JD, Voysey M, D'Emden MC, Laakso M. Baker JR, Kcech AC: FIELD study- investigators. Effect of fenofibrate on amputation events in people with type 2 diabetes mcllitus (FIELD study): a prespecified analysis of a randomised controlled trial. Lancet. 2009:373(9677): 1780-8.

19. Snell EE, Strong FM, Peterson WII. Growth factors for bacteria. VI: Fractionation and properties of an accessory factor for lactic acid bacteria. Biochem $\mathrm{J}$. 1937:31 (10): 1789-1799.

20. Reed LJ, DeBusk BG, Gunsalus IC, Hornbcrger CS Jr. Cristalline alpha-lipoic acid: a catalytic agent associated with pyruvate dehydrogenase. Science. 1951; 114(2952):93-94.

\title{
VASCULAR SURGICAL ASPECT OF DIABETIC FOOT
}

\section{Kostov O. H., $P h D$ Medical University - Plovdiv, Faculty of Social health, assistant DOI: 10.31618/ESU.2413-9335.2019.1.60.42-45}

\section{SUMMARY}

Diabetes continues to grow globally and to spend extremely large resources in the health sector. One of the main factors for the incidence of diabetes is diabetic foot. Necrotic changes in the latter are a complex problem. Ischemia, neuropathy and infection are the three pathological components that lead to complications and often occur together as etiological triad. Neuropathy and ischemia are initiating factors, most often in tandem as neuroischemia until the infection is more a consequence. The role of peripheral arterial disease in diabetic foot long underestimated since typical ischemic symptoms are less common in diabetic patients with ischemia than in nondiabetic patients. Each necrosis diabetic foot must wake suspected vascular disease until proven otherwise. Early referring the patient to a specialist, noninvasive vascular testing, diagnosis and intervention are crucial for the healing of necrosis and for the prevention of amputations, timing is crucial because a window in which we can achieve wound healing and save limbs often can be omitted.

Keywords: diabetic foot, neuroischemia, revascularization, amputation.

Diabetes mellitus and complication are becoming a major cause of morbidity and mortality worldwide. This is a global problem that burdens significant health systems and increased dramatically over the past two decades. For the first time Zmmet in 1992 used the term "diabetes epidemic", whereas as economic burden of disease on society and purely human suffering from it. According to various epidemiological studies cases of diabetes were about 30 million in 1985,177 million in 2000, 285 million in 2010, their number is expected to grow to 360 million in 2030 to a mind-boggling 642 million in 2040. Tap necrosis occurred in $25 \%$ of patients, so it should be given more attention on prevention rather than treatment of ulcers, because five-year mortality rates in diabetic patients who have suffered an amputation of lower limbs, are extremely high, making them more than just lung cancer.
The World Health Organization suggests that in 2030 , diabetes will be the seventh in a row cause of death globally. He one of the leading causes of loss of limb and each year more than 1 million people undergo amputation. Approximately $80 \%$ of these amputations are preceded by foot necrosis. Risk factors for ulcers include neuropathy, peripheral arterial disease, foot deformity, restricted movements in ankle high plantar pressure of the foot, small injuries, ulcers or previous amputations. At least a quarter of these ulcers will heal and to $28 \%$ of them may need some form of amputation.

Diabetic peripheral neuropathy

In diabetic foot neuropathy is the main risk factor for the development of ulcers. According to various sources, the spread between 16 and 66 percent, increasing the duration of diabetes and poor glycemic control. 
Internationally recognized definition of diabetic peripheral neuropathy is the presence of symptoms and / or signs of peripheral nerve dysfunction in diabetic patients after exclusion of other causes.

Neuropathy presents as an inability to capture the temperature difference vibrations, proprioception, pressure, and the most seriously hurt. Some patients have a form of painful sensory neuropathy, which includes symptoms such as burning and tingling - paraplegia. May be affected autonomous, motor and sensory functions of the nerves.

Although many manifestations of neuropathy may remain unrecognized by the patient, the autonomy is perhaps the most neglected in the diabetic foot. The located neuropathy can lead to increased secretion and increase the risk of fungal infections. With the increased rigidity of the skin area parts of friction they become less flexible and thus develop lesions.

The reduction in motor function with neuropathy often affects the inner muscles of the foot and cause joint instability and weakening of muscle groups. Over time, this imbalance leads to flexible deformities that progressively become more rigid. The latter are subjected to a greater pressure and conducive to formation of ulcers.

Screening for peripheral neuropathy includes test feeling of pressure; vibration; measurement of vibration perception threshold; temperature test; test for pain and reflexes.

Obstructive arteriopathy in diabetics is more rapid progression and is usually bilateral. Anatomical features are also typical - more commonly affected vessels below the knee, the changes are symmetrical and multisegment as collaterals, which are generally rare, can also be affected by the process. The nature of the lesions in different parts of the arterial tree of the lower limb is also different. For arteries above the knee characteristic eccentric intraluminar lesions, often with pronounced calcinosis, but there also soft, unstable plaques rich lipid core, often with complications. For vessels below the knee is typical media calcification by increasing it gradually leads to even blockage.

Although the strategy for prevention and treatment of diabetic foot is mainly focused neuropathy, ischemia is the most important factor preventing healing. The combined effect of peripheral neuropathy, and ischemia is defined as neuroischemia. The macroangiopathy and microvascular dysfunction violate perfusion of diabetic foot. Damage to the regulation of blood flow to the opening of arteriovenous shunts and malfunction of precapillary sphincters. Thus blood "bypasses" its recipient, namely capillaries.

Under the leadership of the European Society of Vascular Surgery for diabetic foot ischemia and nevroishemia should be considered together, since both processes may need revascularization.

The clinical picture is rarely symptomatic and most often characterized directly with ischemic lesions or gangrene, which are typical for the later stages of the disease. Poor prognosis in patients with diabetes and necrotic changes of the foot is due to other co-factors such as anatomic distribution, infection, neuropathy, renal insufficiency and involvement of other vascular beds - coronary and cerebrovascular. About 27 percent had disease progression within the next five years, and $4 \%$ experiencing major amputation. $20 \%$ undergo another cardiovascular event (myocardial infarction or stroke).

The prognosis of diabetic patients with critical limb ischemia is even worse, with $30 \%$ of them will undergo major amputation and $20 \%$ will die from another cardiovascular event within a year.

Often patients with diabetes have associated renal insufficiency, which exacerbated their forecast, as the disease is more severe and faster. Renal failure is a major risk factor for ulcers and amputations in these patients, the risk of major amputation in dialysis patients is 4.7 times higher than in non-dialysed.

The presence of peripheral pulses (a. Dorsalis pedis and a. Tibialis posterior) does not exclude the possibility of ischemia of the foot (for example when occlusion on the plangarnus arc), so it is necessary to use other methods for non-invasive examination of the vascular system.

Foot-brachial index is the ratio of arterial tension level ankle to that of the level arm. The test is simple and has a high predictive value for proof. The American Diabete Association offers screening of all patients over 50 years and all insulin-dependent patients of any age in the presence of other cardiovascular risk factors.

Transcutaneous oximetry is used in patients with ulcerations and gangrene, as for normal value taken 50 $\mathrm{mmHg}$.

Duplex ultrasonography allows morphological and functional analysis of the arterial tree.Of the other non-invasive tests most commonly used are kompyutarnotomografskata angiography and magnetic resonance angiography.Primary invasive examination is digital subtraction angiography as used contrast agent may also be a $\mathrm{C} 02$, especially in patients with renal insufficiency. When using standard non-ionic contrast must not forget something very important - for patients treated with metformin latter must be stopped due to the risk of lactic acidosis. The main goal in treatment of the diabetic foot is the prevention of ulceration but in the occurrence of such - quick and adequate treatment of the ulcers. Milestone in addressing diabetic foot is a multidisciplinary approach. According to the strategy of the National Institute for Health and Clinical Excellence team of specialists needed for patients with diabetic foot, consists of a doctor, nurse, trainers, podiatrist, orthotic specialist vascular surgeon, dermatologist , cardiologist, orthopedist, endocrinologist and nutritionist. The American Diabetes Association concluded that the multidisciplinary team reduces the level of amputations by $50-85 \%$ in patients with ulcers.

The main topic of the article is a vascular surgical strategy in the treatment of diabetic foot, but will mention the other components of the therapy:Training the patient - it has been proven that $50 \%$ of the ulcers can be avoided with proper training patients - avoiding risk factors,care for foot, regular checkups, etc.

1. Strict control of blood sugar.

2. Ability to relax the load - include in various orthopedic equipment and surgical correction of deformity of the foot. 
3. Debridement in necrotic changes - surgical (sharp), mechanical (hydrotherapy, dry dressing), autolysis, enzyme (streptokinase), biologically (sterile larvae).

4. Dressings for diabetic ulcers - hydro colloids, hydrogels, foam, film dressings, alginates, impregnated with silver.

5. Others - hyperbaric oxygenation, electrostimulation therapy, negative pressure, growth factors, skin grafts and other.

6. Aggressive treatment of an infection.

The combination of ASA $100 \mathrm{mg}$ and a low dose of rivaroxaban $2 \times 2.5 \mathrm{mg}$ (COMPASS study) proves to be extremely suitable in patients with stable atherosclerotic pathology.

The main goal in vascular surgical treatment of diabetic foot is the direct revascularization and restore perfusion to the foot and toes. This is achieved through three main methods: endovascular, open or hybrid surgery.The success of vascular reconstruction of any kind depends on a free distal vessel and stored plantar arch. However, it is shown that up to $20 \%$ of patients with successful reconstructions do not achieve healing of ulcers. This shows the complexity of the problem, especially microcirculations dysfunction. Therefore develop different methodologies for assessing perfusion of the limb before and after revascularization, they can be differentiated patients who would have really benefited from the intervention. So far the indications for revascularization are:

1. limit claudication and / or pain at rest

2. trophic lesion that shows no signs of healing after one month of adequate treatment.

These techniques are mini-invasive, patients tolerate them lighter and has the possibility of repetition of procedures. Naturally patency is significantly shorter than the distal vascular bypass operations, which are considered the gold standard, but that patients with many comorbidities are particularly suitable and friendly.More common are cases where patients are referred for endovascular treatment after unsuccessful surgery that. Endovascular treatment does not preclude open surgery, it must still be applied by professionals who know or at least have seen distal bypass operations, in order not to violate local, typical of anastomoses. It has been shown that open surgery is much more difficult after a failed endovascular treatment, more frequent are complications. The opposite is true with the same success and open surgery - you need to keep the vessels of extremities despite being occluded, not ligated or resected since then endovascular treatment is impossible.Hybrid surgery using the best of both worlds - often composed of local or remote arteries and endovascular balloon part with angioplastic and / or implantation of the stack.

Due to the nature of the disease open surgery relies mostly bypass operations to small vessels of the lower leg and foot, so-called distal bypass. Main conduit in these operations is the autovein. Best results are achieved when three factors are present: adequate inflow, good vein and stock ran off. When missing or damaged vein may be used a prosthesis or combination of prosthesis / vein (composite bypass), but with significantly lower primary patency than adequate in size autovena.To determine which method of treatment to choose, it is important to consider four main factors:

1. Clinical status of patients - age, comorbidity, life expectancy, type of anesthesia and others.

2.Evaluation of foot - good for a bypass, there infection, necrosis, affecting places anastomoses and others.

3. Quality of venous conduit - assessment of all veins that can be used for graft.

4. Recipient vessel - size, calcification, theory of wounds, etc. The goal of revascularization is to provide direct blood flow to the foot, possibly via an anterior and / or posterior tibial artery, and for failure - and to the peroneal artery.

More serious is the situation when there is no recipient court, distal run off and compromised microcirculation of the foot called desert foot. In such cases, we have four options:

1. May try conservative treatment with prostaglandins and other vasodilators (with unproved effect), the application of stem cells and other growth factors;

2.Arterial bypass reconstruction to shlateralen court - a labor-intensive operation with uncertain effect;

3.Venous arterializatsiya - the idea is very old was first mentioned back in 1896 by Francois Franck M., and was applied in 1912 by Halstead and Vaughan to save limbs from gangrene.

In the past, the main problem was not so much surgical performance than the destruction of venous valves. As is known valves increase in number from the proximal to distal direction of the limbs, each posterior tibial vein has valves $8-19$, and each front tibial or peroneal - 8-11. The aim is to provide blood flow to a level metatarsal and digital veins.

Known are two modern methods for such performance: endovascular and hybrid.Endovascular treatment - the idea of the invention is the following: used two ultrasonic catheter (venous and arterial), and are positioned side by side at the distal passable artery. Then both catheter made A-B fistula, which is covered with the standgraft. In this way we receive flow from the artery to the vein. It should dismantle the valve apparatus endovascular valve and verification of the results. The results are more than impressive with saving the limb in more than $71 \%$.

Hybrid procedure - as yet do not have free with Lim Flow, another way of deep vein arterialization is the hybrid approach. The technique consists of the following components: performing operational A-B fistula through the bypass of the distal accessible artery (which may be of the common femoral artery) to the deep crural vein. So created $A B$ fistula will redirect blood flow from the graft to the proximal veins. To be effective the procedure will be needed and endovascular part - removing the valves in a distal direction.

Regardless of which of the two methods is used, deep vein arterialization gives definitive solution for revascularization in these otherwise "doomed" amputation patients. 
4. Amputations - emergency or healing. The indications for amputation include removing infected or gangrenous tissue and creating functional foot or stump to take the boot or prosthesis respectively.

Conditional amputations are divided into small fingers, metatarsus and large - transtibial and transfemoral. Generally speaking, the more proximal amputation is, the more complications. During the first 12 months after the amputation of a finger amputation risk of new $22.8 \%$ ipsilateral and contralateral $3.5 \%$. For 5 years, the risk becomes respectively $52.3 \%$ and $29.5 \%$. There is no direct link between amputations and deaths, but several studies have shown a very strong bond between them. Diabetic patients have a 55 percent higher risk of death after amputation than non-diabetics. The one-year mortality after amputation of the finger $6.6 \%$ after amputation of the part of the sole 10,5\% and $18,2 \%$ following a major amputation. The five-year mortality following a major amputation is about $36 \%$. The risk of amputation is determined most exactly with WIFI (Wound, Ischemia, and Foot Infection) American Society of Vascular Surgery.

\section{Conclusion}

The incidence of diabetes increases significantly and diabetic foot and its complications continue to be a major challenge to the health system and in particular to vascular specialists. The neuroishemic diabetic foot is much more common than believed. From a practical point of view, patients with ischemic clean and neuroishemic pathology should be treated in the same way. Each wound in diabetic patient should be viewed primarily as ischemic until proven otherwise. Any patients with these findings need to be referred for consultation with a vascular specialist, because any delay can be detrimental to the limb (the time is tissue!). Treatment of diabetic foot depends on two factors: it is essential that the patient is sufficiently informed and involved with their disease and treatment by specialists to be multidisciplinary. A combination of these two factors chance diabetics to avoid or at least away significant complications of the diabetic foot is very large. Preventing large and possibly a small amputation, we "save" largely patient's life.

\section{References:}

1. Bostrom P. Wu.J. Jedrychowske MP, et al A PGCI- $\alpha$-dependent myokine that drives brown-fat-like development of white fat ana thsmiogenesis. Nature. 2012;481:463-468

2. Conway B, Mille RG. Costacou T. ei al. Adiposity and mortality in type 1 diabetes. Int Obes (Loud). 2009;33:796-805

3. Costacou T, Evans RW, Orchard TJ. Highdensity lipoprotein cholesterol in diabetes: is higher always better? J Clin Lipidol. 2011:5:387-394.

4. Costacon T, Ferrell RE, Orchard, J. Haptoglobin genotype: a determinant of cardiovascular complication risk in type 1 diabetes. Diabetes. 2008;57:17021706.

5. Dabelea D, Kinney G, Snell-Bergeon JK. Hokanson JE. Eckel RH, Ehrlich J, Garg S, Hamman RF. Rewers M. Coronary Artery Calcification in Type i Diabetes Study. Effect of type I diabetes on the gender difference in coronary artery calcification: a role for insulin resistance" The Coronary Artery Calcification in Type 1 Diabetes (CACTI) Study. Diabetes. 2003;52(11):2833-2839.

6. Darabian S, Backland JY. Cleary PA. Sheidaec N, Bebu I, Lachin JM. Bndoff MJ; DCCT/EDIC Research Group. Significance of epieardial and intrathoracic adipose tissue volume among type 1 diabetes patients in the DCCPEDIC: A pilot study. PLoS One. 2016: 11(7):e0159958.

7. De Ferranti SD, de Boer IH, Fonseca V. et. al. Type 1 Diabetes Mellitus and Cardiovascular Disease: A Scientific State- ment From the American Hears Association and American Diabetes Association. Diabetes Care. 2014:37.2843-2863.

8. Du Bose SM Flermann JM. Tamboriane WV, Beck RW, Dost A. DiMeglio LA, Schwab KO, Holl RW, Hofer SE Maahs DM; Type 1 Diabetes Exchange Clinic Network and Diabetes Prospective Follow-up Registry. Obesity in Youth with Type 1 Diabetes in Germany, Austria, and the United States. J Pediatr 2015 Sep;167(3):627-32.c 1-4.

9. Tapes D, Lao J, Carlssoo PO. Increased levels of irisin in people with long-standing Type 1 diabetes. Diabet Med. 2015 Sep;32(9): 1172-76. 